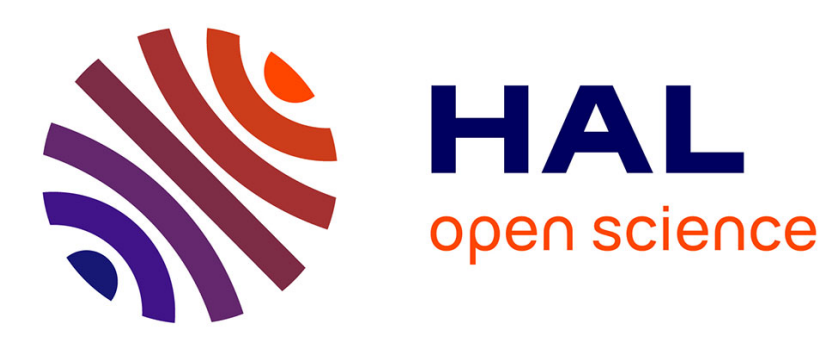

\title{
On the behaviour of the ANM continuation in the presence of bifurcations
}

Sébastien Baguet, Bruno Cochelin

\section{To cite this version:}

Sébastien Baguet, Bruno Cochelin. On the behaviour of the ANM continuation in the presence of bifurcations. Communications in Numerical Methods in Engineering, 2003, 19 (6), pp.459-471. 10.1002/cnm.605 . hal-00088346

\section{HAL Id: hal-00088346 https://hal.science/hal-00088346}

Submitted on 24 Jul 2017

HAL is a multi-disciplinary open access archive for the deposit and dissemination of scientific research documents, whether they are published or not. The documents may come from teaching and research institutions in France or abroad, or from public or private research centers.
L'archive ouverte pluridisciplinaire HAL, est destinée au dépôt et à la diffusion de documents scientifiques de niveau recherche, publiés ou non, émanant des établissements d'enseignement et de recherche français ou étrangers, des laboratoires publics ou privés.

\section{(c)(1)}

Distributed under a Creative Commons Attribution| 4.0 International License 


\title{
On the behaviour of the ANM continuation in the presence of bifurcations
}

\author{
S. Baguet and B. Cochelin \\ Laboratoire de Mécanique et d'Acoustique, UPR CNRS 7051, Ecole Supérieure de Mécanique de Marseille \\ IMT-Technopôle de Château Gombert, 13451 Marseille Cedex 20, France
}

The asymptotic-numerical method (ANM) is a path following technique which is based on high order power series expansions. In this paper, we analyse its behaviour when it is applied to the continuation of a branch with bifurcation points. We show that when the starting point of the continuation is near a bifurcation, the radius of convergence of the power series is exactly the distance from the starting point to the bifurcation. This leads to an accumulation of small steps around the bifurcation point. This phenomenon is related to the presence of inevitable imperfections in the FE models. We also explain that, depending on the maximal tolerated residual error (out-of-balance error), the ANM continuation may continue to follow the fundamental path or it may turn onto the bifurcated path without applying any branch switching technique.

KEY WORDS: continuation; asymptotic-numerical-method; bifurcation; series convergence

\section{INTRODUCTION}

The asymptotic-numerical-method (ANM) is an alternative to the classical Newton-Raphson techniques [1] for making the continuation of a non-linear solution with respect to a parameter, typically in structural mechanics, for tracing an equilibrium path with respect to a loading parameter [2-4]. The basic principle of the ANM continuation is to determine the path by a succession of high order power series expansions (perturbation method) with respect to a well chosen path parameter. It is in fact a high order 'continuous' predictor without any correction. Because few stiffness matrix decompositions are required, only one per step, the performances as regard to computing time are attractive. However, the very advantage of the ANM over incremental-iterative procedure is that it yields an analytic continuous representation of the path, which provides significant benefits for the continuation. For instance, the step-length 
has not to be estimated in advance and, if necessary, to be adjusted according to the convergence behaviour of the corrector. It is always determined after the computation of the power series by simply analysing their radius of convergence. Using this crucial information, the design of robust continuation algorithms with automatically determined optimal step-length is easy.

When the path has a bifurcation point, the ANM continuation has a very specific behaviour which is not encountered with classical predictor-corrector algorithms (Newton-Raphson for instance). As the current step of continuation approaches the bifurcation, the step-length is automatically severely reduced. There is an accumulation of small steps and everything looks as if the continuation process 'knocks' against the bifurcation. Depending on the residual error tolerance, the continuation algorithm may continue to follow the fundamental branch, but it may also switch to the bifurcated branch. The main objective of this paper is to explain this step accumulation and the reason for the switch to the bifurcated branch. It is not actually a proposition towards a branch switching algorithm, we limit ourselves to examine the behaviour of the ANM continuation in the presence of a bifurcation.

The basic ANM has already been presented in a number of full-length papers dedicated to several applications [5-10]. Here, we begin with a minimal review of the ANM continuation, before embarking on the detail of the continuation of a path with a bifurcation.

\section{A MINIMAL REVIEW OF THE ANM CONTINUATION}

Let us consider a non-linear problem depending on the unknown $u$ and on a parameter $\lambda$. Let

$$
R(u, \lambda)=0
$$

be the governing equations of this non-linear problem. The principle of the ANM continuation consists in describing the solution path by computing a succession of truncated power series expansions of the following form:

$$
\begin{aligned}
& u(a)=u_{0}^{j}+a u_{1}+a^{2} u_{2}+a^{3} u_{3}+\cdots+a^{N} u_{N} \\
& \lambda(a)=\lambda_{0}^{j}+a \lambda_{1}+a^{2} \lambda_{2}+a^{3} \lambda_{3}+\cdots+a^{N} \lambda_{N}
\end{aligned}
$$

where $\left(u_{0}^{j}, \lambda_{0}^{j}\right)$ is a given starting point on the path, $N$ the order of truncature, and $a$ a path parameter (the classical arc length parameter for instance). By using a high order of truncature, say from 10 to 30 , the series provide an accurate local continuous representation of the solution path. Obviously, such series generally have a finite radius of convergence, and they can give only a limited part of the path. Also, it becomes necessary to use several expansions from successively updated starting points to describe the full path as shown in Figure 1.

Hence, a crucial point of the ANM continuation is to analyse the range of validity of each series expansion in order to define a suitable end point that will serve as a new starting point for the next step of continuation. We define this range of validity of the series as the interval $\left[0, a_{m}\right]$ of the path parameter for which the series satisfy the accuracy requirement $\|R(u(a), \lambda(a))\| \leqslant \varepsilon_{R}$, where $\varepsilon_{R}$ is the tolerance error for $R$ (maximal out-of-balance forces in 


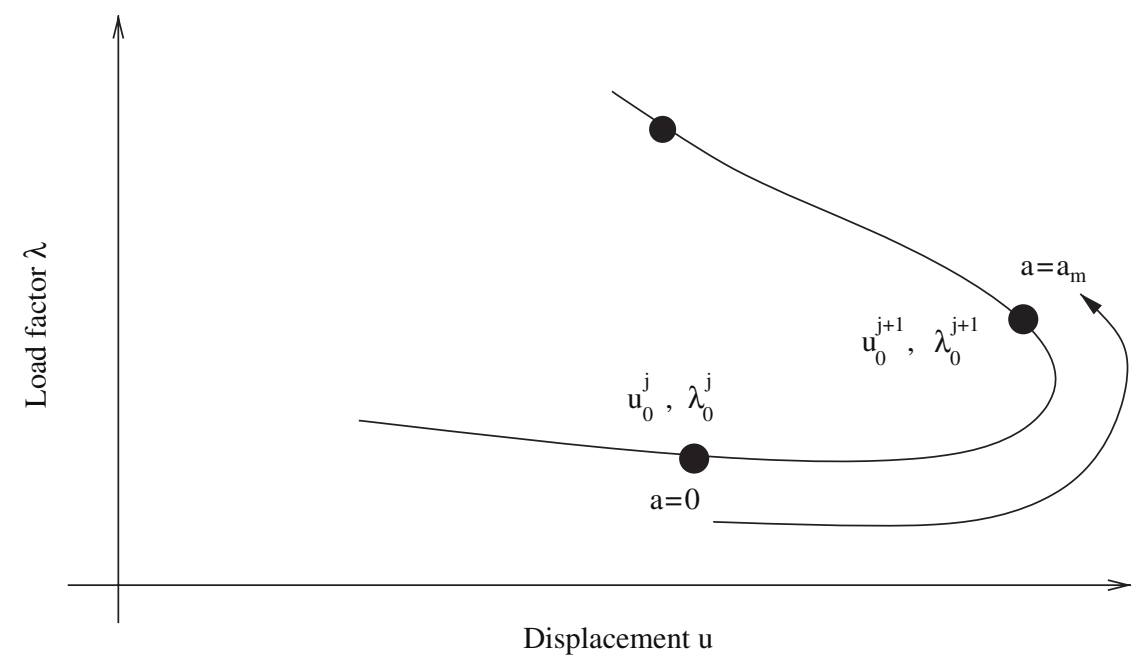

Figure 1. Principle of continuation with the ANM.

structural mechanics). Here, we shall use an asymptotic expansion of $R$ in order to determine this range of validity, i.e., to compute the value of $a_{m}$. By reporting the truncated series expansions (2) into the governing equation (1) and ordering like powers of the expansion parameter $a$, we get

$$
R\left(u_{0}^{j}+a u_{1}+\cdots+a^{N} u_{N}, \lambda_{0}^{j}+a \lambda_{1}+\cdots+a^{N} \lambda_{N}\right)=R_{0}+a R_{1}+a^{2} R_{2}+\cdots+a^{N} R_{N}+a^{N+1} R_{N+1}+\cdots
$$

The first term $R_{0}=R\left(u_{0}^{j}, \lambda_{0}^{j}\right)$ is null since the starting point $\left(u_{0}^{j}, \lambda_{0}^{j}\right)$ is on the path. Since the series are truncated at order $N$ and according to the perturbation principle, all the terms $R_{k}$ with $k=1, \ldots, N$ are also null. Indeed, $R_{k}=0$ corresponds to the linear problem that has to be solved at order $k$ in order to obtain $u_{k}$ and $\lambda_{k}$. As a result, the first non-zero term of the $R$ expansion (3) is $a^{N+1} R_{N+1}$, where $R_{N+1}$ is simply the r.h.s. vector of at order $k+1$ (easy to compute [11]). In practice, a very good approximation for $R$ is obtained by keeping this first term and neglecting all the other higher order terms. Hence, the requirement that the norm of $R(u(a), \lambda(a))$ is below $\varepsilon_{R}$ leads to the following maximal value for the path parameter $a$.

$$
a_{m}=\left(\frac{\varepsilon_{R}}{\left\|R_{N+1}\right\|}\right)^{\frac{1}{N+1}}
$$

So, for $a \in\left[0, a_{m}\right]$, the series (2) give an analytical continuous representation of the branch which satisfies the accuracy requirement $\|R(a)\| \leqslant \varepsilon_{R}$ everywhere. The last point of the interval $\left(u\left(a_{m}\right), \lambda\left(a_{m}\right)\right)$ serves as the new starting point $\left(u_{0}^{j+1}, \lambda_{0}^{j+1}\right)$ for the next step of continuation. Practically, this continuation algorithm requires only two parameters: the order of truncature $N$ and the error tolerance $\varepsilon_{R}$ which defines the accuracy of the solution. The size of the steps 


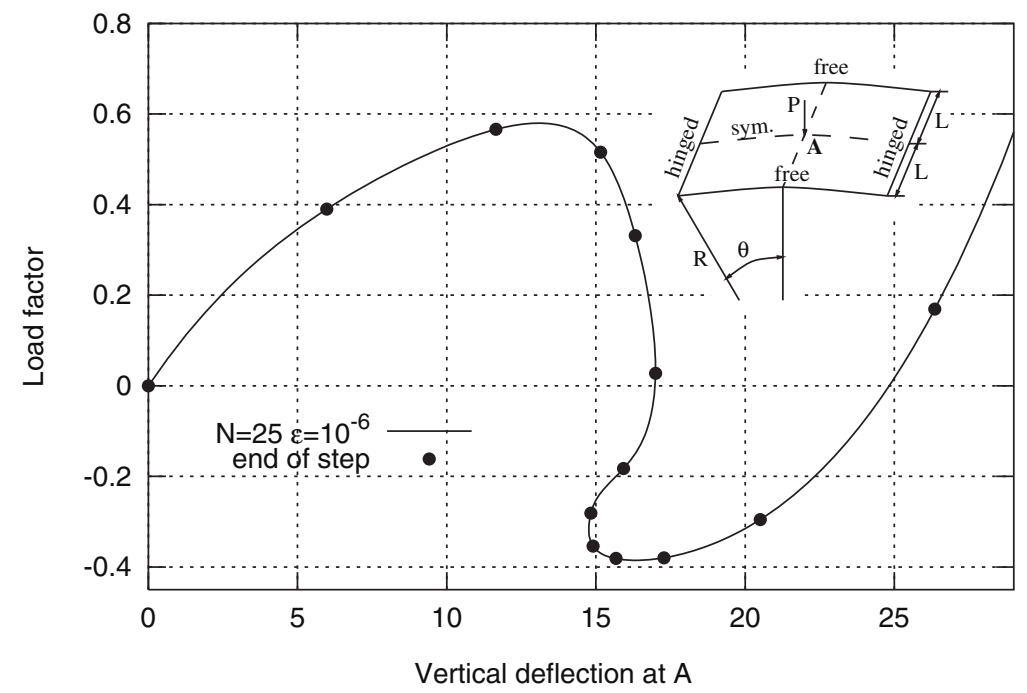

Figure 2. Continuation of the path with no bifurcation $\left(N=25\right.$ and $\left.\varepsilon_{R}=10^{-6}\right), R=2540 \mathrm{~mm}$, $L=254 \mathrm{~mm}, h=6.35 \mathrm{~mm}, \theta=0.1 \mathrm{rad}, E=3102.75 \mathrm{Mpa}, v=0.3, P=1000 \mathrm{~N}$.

are automatically given by the criterion (4). The user of the algorithm has only to specify how many steps he (she) wants to do.

\section{EXAMPLES OF ANM CONTINUATION}

\subsection{Path without bifurcation}

We consider for this the well-known benchmark problem of a thin simply supported cylindrical shell subjected to a central point load as described in Reference [4, p. 268]. By meshing only one quarter of the shell and applying suitable symmetry conditions on the edges, the solution path has no bifurcation point. At order $N=25$, and with a tolerance $\varepsilon_{R}=10^{-6}$, it is described in 12 steps as can be seen in Figure 2.

\subsection{Path with bifurcations}

By meshing one half of the shell instead of one quarter, the response is now made of the same fundamental path as above, and of a secondary closed path that corresponds to a buckling with a symmetry breaking. The two paths are connected at two bifurcation points as shown in Figure 3. Using the same parameter $N=25$ and $\varepsilon_{R}=10^{-6}$, the ANM continuation has now a rather different behaviour. After a first step which is almost identical to the first step in the previous example, the algorithm makes a shorter second step, with an end point which is just behind the bifurcation point. The third and the fourth steps appear to be short, the length of the fourth step being the double of the third one. Once the updated starting point is far from the bifurcation, the step length retrieves the same behaviour as in the case without bifurcation. Finally, we can see that the same phenomenon of small steps accumulation also occurs behind 


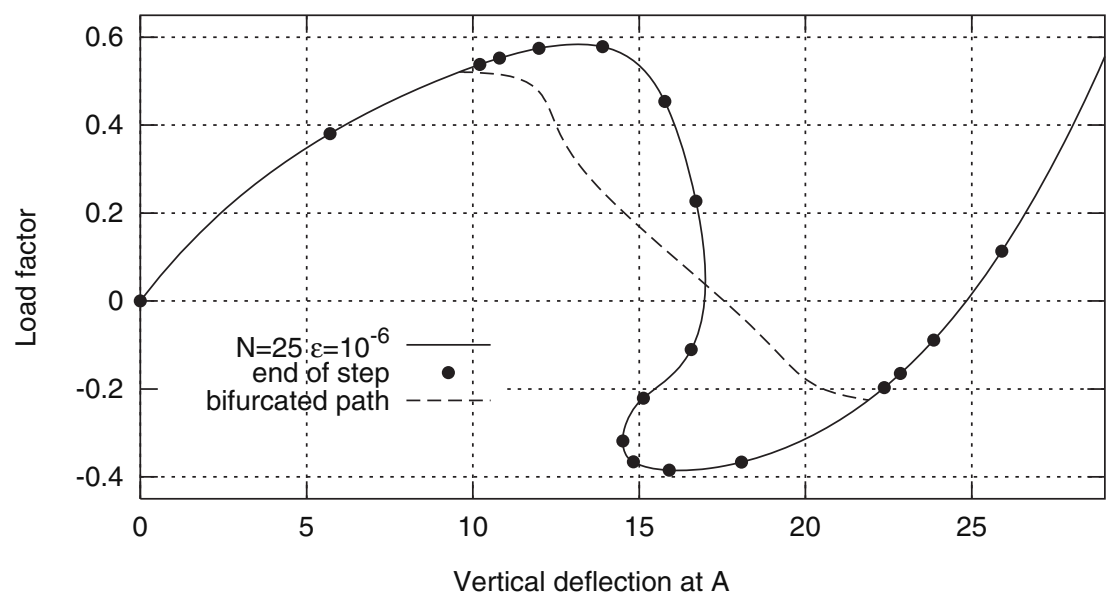

Figure 3. Continuation of the path with bifurcations, $N=25$ and $\varepsilon_{R}=10^{-6}$. The bifurcated branch is plotted with dashed lines. Small steps occur behind the bifurcations.

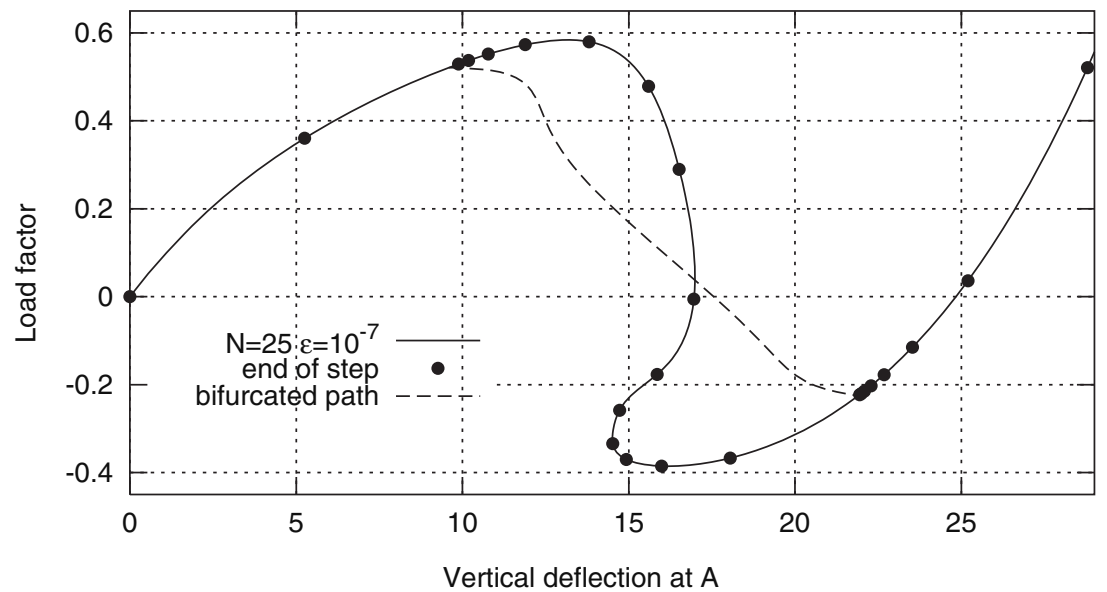

Figure 4. Path with bifurcations. With a smaller tolerance $\varepsilon_{R}=10^{-7}(N=25)$ the step accumulation is more pronounced.

the second bifurcation point. If we now reduce the tolerance criterium $\varepsilon_{R}$ to $10^{-7}$, the step accumulation is more pronounced (see Figure 4). The end of the second step gets closer to the bifurcation, and more steps are needed to run away from the bifurcation. Here, the third, fourth and fifth steps are short, and there is again a doubling of step length between the third and fourth steps, and between the fourth and fifth steps. Finally, if we take $\varepsilon_{R}=10^{-8}$, the end of the second step is now before the bifurcation point. A severe accumulation occurs and the continuation algorithm turns onto the bifurcated path instead of following the fundamental one as shown in Figure 5. 


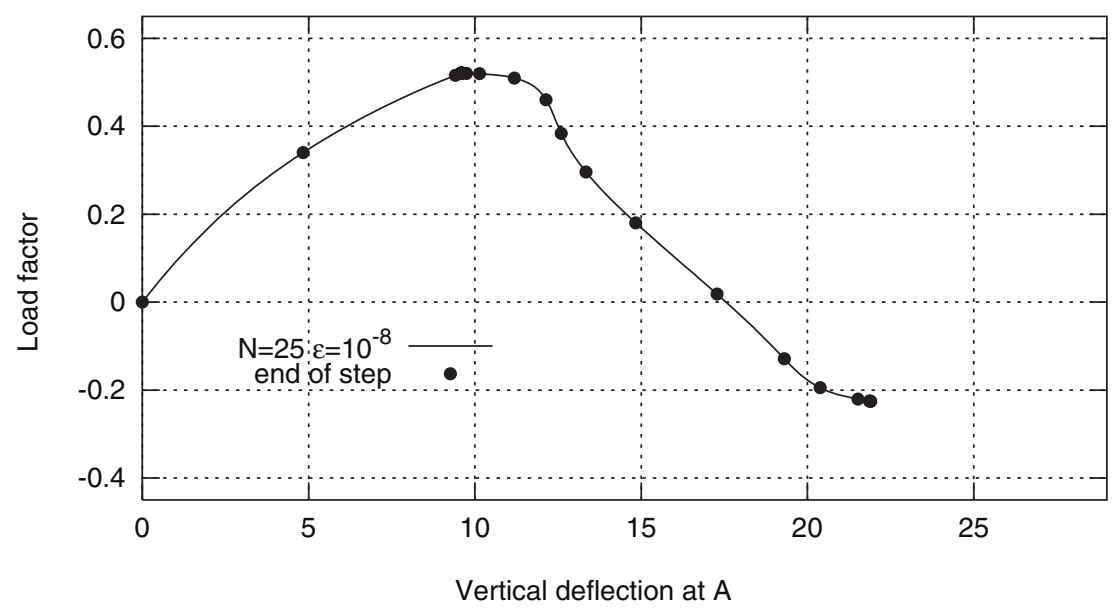

Figure 5. Path with bifurcations. With $\varepsilon_{R}=10^{-8}(N=25)$ the algorithm turns onto the bifurcated path.

This behaviour, with an accumulation of short steps and possibly a turn onto the bifurcated branch, is very specific of the ANM continuation. It is not encountered with predictor-corrector methods. For instance, the classical Newton-Raphson method with prescribed step length generally gives exactly the same results for the two tests used above. Nevertheless, the test with bifurcations can make troubles for the corrector if the end of a step is very near the bifurcation. In this case, an increase of iterations and a jump onto the bifurcated branch can occur. For the same reason, Newton-Raphson methods with self adaptative step length $[4,12]$ can behave differently when applied to the two tests, but the differences are not as pronounced as in the case of the ANM continuation.

\section{THE STEP ACCUMULATION AND THE TURN ONTO THE BIFURCATED BRANCH}

To explain the step accumulation and the reason why the algorithm stays on the fundamental branch or turns onto the bifurcated one, we shall consider two very simple examples, with one and two d.o.f., and for which analytical solutions are known.

The first example concerns a vertical rigid bar hinged at its base and connected to a spiral spring that supplies a linear restoring moment (see Figure 6). The bar is loaded by an almost perfectly vertical force. We denote by $u$ the angle of rotation of the bar, $\lambda$ and $\eta \lambda$ the vertical and horizontal components of the force, where $\eta$ is a small imperfection parameter. The balance of momentum leads to the (dimensionless) governing equations

$$
R(u, \lambda)=(1-\lambda) u-\eta \lambda=0
$$

In the perfect case $\eta=0$, the solution is made of two straight branches $u=0$ and $\lambda=1$, whereas in the imperfect case $\eta \neq 0$, it is made of two hyperbolae $u=\eta(\lambda /(1-\lambda))$. 


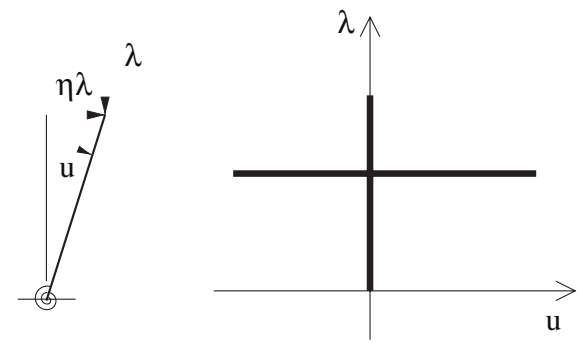

Perfect case $\eta=0$

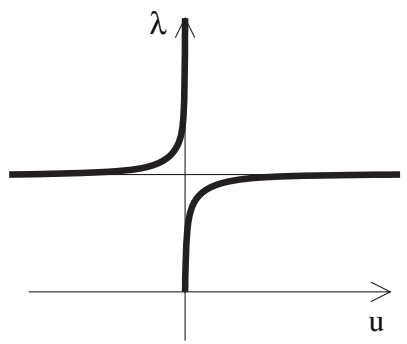

Imperfect case

Figure 6 . The rotating rigid bar under vertical load.

Let us apply the ANM continuation from the origin $(u=0, \lambda=0)$ by taking the load parameter $\lambda$ as the path parameter. Hence, we seek

$$
u(\lambda)=u_{0}+\lambda u_{1}+\lambda^{2} u_{2}+\cdots
$$

Introducing this expansion into the governing equation (5) and equating like powers of $\lambda$ gives $u_{i}=\eta$ for $i=1, \ldots, N$, that is

$$
u(\lambda)=\lambda \eta+\lambda^{2} \eta+\cdots
$$

which is nothing else than the truncated Taylor expansion of the exact solution $u=\eta(\lambda /(1-\lambda))$.

In the perfect case $\eta=0,(7)$ provides $u=0$ which is the exact solution. The range of validity of the series is infinite. This is not the case anymore when there is an imperfection $\eta \neq 0$, whatever the smallness of $\eta$. Indeed, the radius of convergence of the power series (7) given by $\lim _{n \rightarrow+\infty} u_{n} / u_{n+1}$ is equal to one. It is exactly the distance measured with $\lambda$ from the starting point to the bifurcation point. So, we can say that the imperfection makes the bifurcation 'visible'. Let us analyse the quality of the series (7) truncated at order $N$. The exact expression of the residual is

$$
\left|R\left(\lambda \eta+\lambda^{2} \eta+\ldots \lambda\right)\right|=\eta \lambda^{N+1}
$$

It is a continuous increasing function of $\lambda$ which grows rapidly for $\lambda>1$ when $N$ is large. Here, the criterion (4) that gives the range of validity of the series yields

$$
\lambda_{m}=\left(\frac{\varepsilon_{R}}{\eta}\right)^{\frac{1}{N+1}}
$$

So, the step length depends on the ratio between the residual tolerance $\varepsilon_{R}$ and the imperfection $\eta$. The end point of the first step of continuation will be behind the (quasi-)bifurcation if $\varepsilon_{R}>\eta$ and before the bifurcation otherwise. As we shall see later on, this makes the decision for the next step of continuation to follow the fundamental branch or to turn onto the bifurcated one. Notice that if $N$ is very large, the end point will always be closed to the bifurcation.

Let us now consider a second example in order to complete the analysis. It consists of two truss elements loaded in a plane by a vertical force $\lambda$ and a small horizontal one $\eta \lambda$ (see 


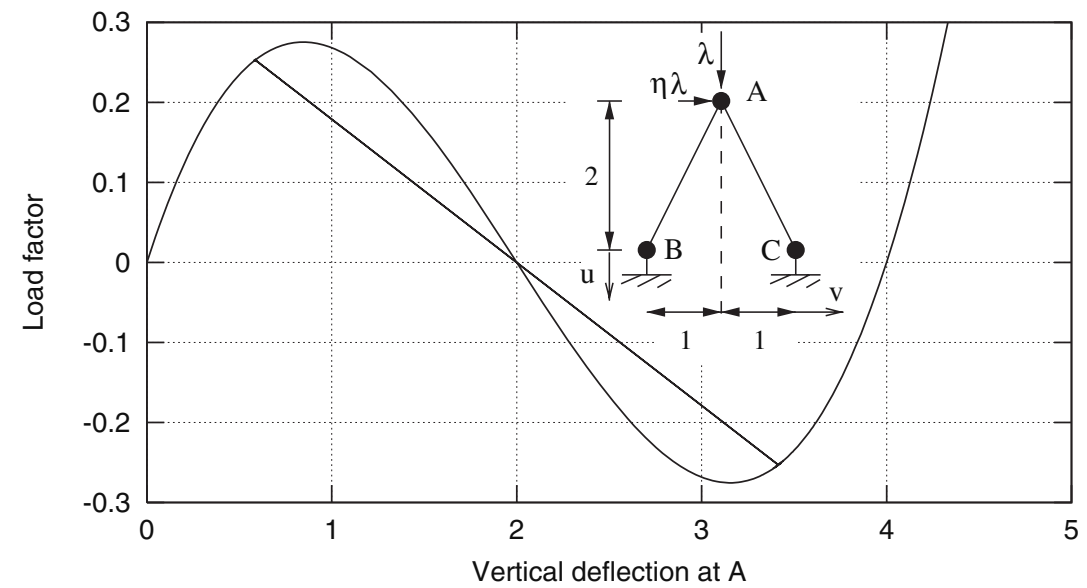

Figure 7. Data and load/deflection curves of the truss model. On the fundamental path the horizontal displacement $v$ of point $\mathrm{A}$ is always zero (symmetric solution). The secondary closed loop path correspond to a non-symmetric solution where the two bars go together to the left or to the right.

Figure 7). The degrees of freedom are $u$ and $v$, the vertical and the horizontal displacements of the loaded node. The forces in the truss are taken to be proportional to the Green strain elongation $e=\frac{1}{2}\left(l^{2}-l_{0}^{2}\right) / l_{0}^{2}$ as in Reference [4].

Taking a tension stiffness equals to one, the potential energy of the system is

$$
P=\frac{1}{2} l_{0} e_{1}^{2}+\frac{1}{2} l_{0} e_{2}^{2}-\lambda u-\eta \lambda v
$$

and the equilibrium equations are

$$
R(u, v, \lambda)=\left\{\begin{array}{l}
\frac{\partial P}{\partial u}=\frac{1}{5 \sqrt{5}}\left(u(u-4)+v^{2}\right)(u-2)-\lambda=0 \\
\frac{\partial P}{\partial v}=\frac{1}{5 \sqrt{5}}\left(10-4 u+u^{2}+v^{2}\right) v-\eta \lambda=0
\end{array}\right.
$$

For the perfect case $\eta=0$, the solution is made of a fundamental branch that satisfies $v=0$, $\lambda=(1 / 5 \sqrt{5}) u(u-2)(u-4)$, and of a closed loop branch that is connected to the fundamental one at two bifurcation points. On the fundamental branch the tangent stiffness matrix $(2 \times 2)$ is diagonal since $\partial^{2} P / \partial u \partial v=0$, and its determinant $\left(\partial^{2} P / \partial u^{2}\right)\left(\partial^{2} P / \partial v^{2}\right)$ vanishes for $u=2 \pm \frac{2}{3} \sqrt{3}$ corresponding to the limit points with respect to $\lambda$, and for $u=2 \pm \sqrt{2}$ corresponding to the bifurcation points.

For this example the suitable path parameter is the vertical displacement $u$. Thus, we take $a=u$ and the series expansions for $\lambda$ and $v$ become

$$
\begin{aligned}
& \lambda(u)=\lambda_{0}+\lambda_{1} u+\lambda_{2} u^{2}+\lambda_{3} u^{3}+\cdots \\
& v(u)=v_{0}+v_{1} u+v_{2} u^{2}+v_{3} u^{3}+\cdots
\end{aligned}
$$


Table I. Coefficients of the series in the perfect and imperfect cases.

\begin{tabular}{lll}
\hline Coefficients & Perfect case $\eta=0$ & Imperfect case $\eta=10^{-8}$ \\
\hline$\lambda_{1}=$ & +0.71554175 & +0.71554175 \\
$\lambda_{2}=$ & -0.53665631 & -0.53665631 \\
$\lambda_{3}=$ & $+0.89442719 \mathrm{E}-0$ & $+0.89442719 \mathrm{E}-01$ \\
$\lambda_{4}=$ & 0. & $-0.23726984 \mathrm{E}-14$ \\
$\lambda_{5}=$ & 0. & $-0.45347614 \mathrm{E}-14$ \\
$\lambda_{6}=$ & 0. & $-0.86535831 \mathrm{E}-14$ \\
$\lambda_{7}=$ & 0. & $-0.16330004 \mathrm{E}-13$ \\
$\ldots$ & & $\ldots$ \\
$\lambda_{22}=$ & 0. & $-0.12095001 \mathrm{E}-09$ \\
$\lambda_{23}=$ & 0. & $-0.21457631 \mathrm{E}-09$ \\
$\lambda_{24}=$ & 0. & $-0.38013517 \mathrm{E}-09$ \\
$\lambda_{25}=$ & 0. & \\
\hline
\end{tabular}

In the perfect case $(\eta=0)$, the introduction of these series into (11) and the identification of the coefficients $\lambda_{i}$ and $v_{i}$ leads to the exact fundamental solution

$$
\begin{aligned}
& \lambda(u)=\frac{8}{5 \sqrt{5}} u-\frac{6}{5 \sqrt{5}} u^{2}+\frac{1}{5 \sqrt{5}} u^{3} \\
& v(u)=0
\end{aligned}
$$

at the first step of the ANM continuation. The range of validity of the series is infinite.

Let us now introduce a small imperfection $\eta=10^{-8}$ in the model. In that case, the analytic solution is not known and the hand computation of the series coefficients becomes tedious. We use our ANM-oriented FEM code to compute the series (12) up to order 200. The result is that the coefficients of the $\lambda$ series are only slightly modified by the imperfection as it is shown in Table I. However, the radius of convergence of the series is largely modified. Indeed, using a Domb-sykes plot [13], the limit of $\lambda_{N} / \lambda_{N+1}$ do seems to be $2-\sqrt{2}=0.5857664$ (see Figure 8). Once again this corresponds exactly to the distance measured with the path parameter $u$ from the starting point to the bifurcation point.

To analyse the quality of the truncated series, we have numerically determined the evolution of norm of the residual error $R$ of (11) with respect to the path parameter $u$ for various order of truncature $N$ of the series (12) . The results are reported using logscale in Figure 9. We can see that the plots are almost straight lines, which is in agreement with the approximation $R \simeq a^{N+1} R_{N+1}$. The three lines cross each other for $u \simeq 2-\sqrt{2}$. Before that value of $u$, the quality of the solution increases with $N$ (convergence), and after that value, it decreases (divergence). This confirms that $2-\sqrt{2}$ is the radius of convergence of the series.

According to the criterion (4) the value $a_{m}$ of the path parameter at the end point of the step is given by the intersection of the horizontal line corresponding to the required tolerance with the residual curves. Once again, we can see that the end point will be behind or before the bifurcation depending on the value of the tolerance error $\varepsilon_{R}$. For $\varepsilon_{R}=10^{-6}$, the step length $a_{m}$ will be always greater than $2-\sqrt{2}$, and the end point always behind the bifurcation, whatever the order $N$. Here, the algorithm follow the fundamental branch as shown in Figure 10. For $\varepsilon_{R}=10^{-10}, a_{m}$ is smaller than $2-\sqrt{2}$ and the end point is always before the bifurcation. In 


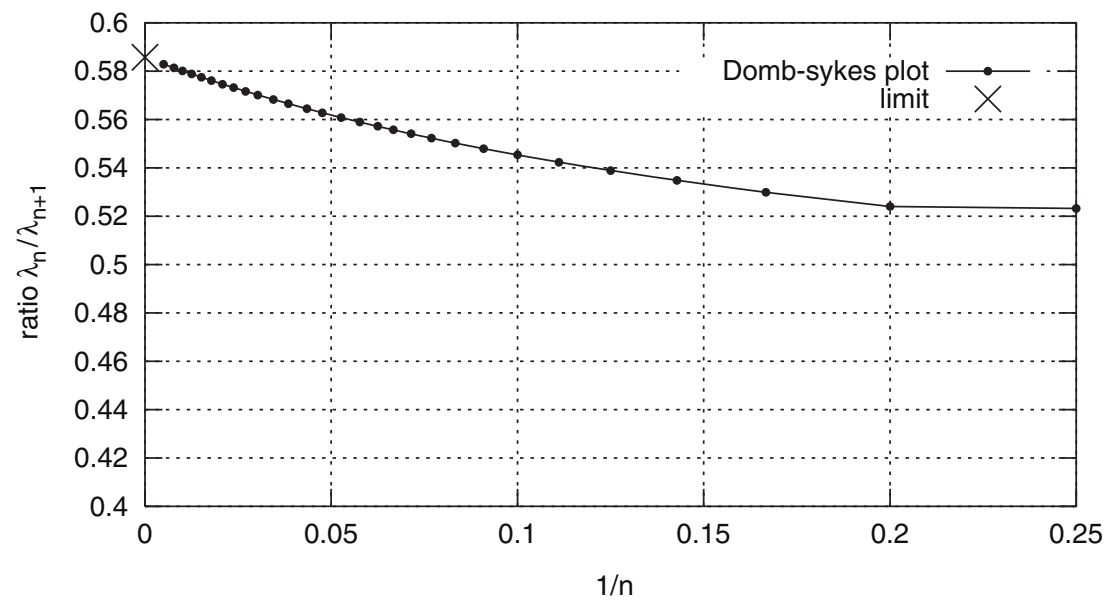

Figure 8 . Domb-sykes plot of the $\lambda$ series. The limit of the curve indicates the radius of convergence.

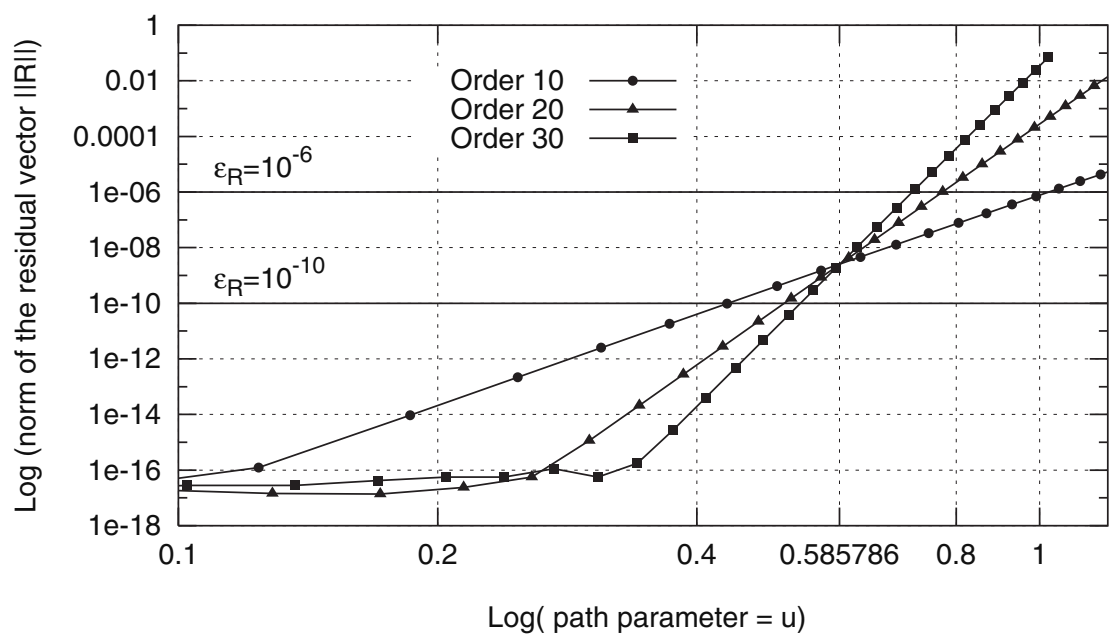

Figure 9. Evolution of the residual error of the truncated series with the path parameter $u$ for three orders of truncature 10,20 and 30.

that case, the next step of continuation gives an accurate description of the sharp turn of the branch and the bifurcated branch is followed, as shown in Figure 11. This can occur only if the required precision $\varepsilon_{R}$ is below the level of the imperfection that is responsible for the transfer of the pure bifurcation into a quasi-bifurcation.

With these results in mind, we are now able to examine the behaviour of the ANM continuation on the cylindrical shell benchmark of Section 3. Because of limited accuracy in the data, for instance in the position of the nodes ( 8 decimal digits in the data file), the symmetry is not perfectly satisfied within the FEM model. As a consequence, there are no pure bifurcations on 


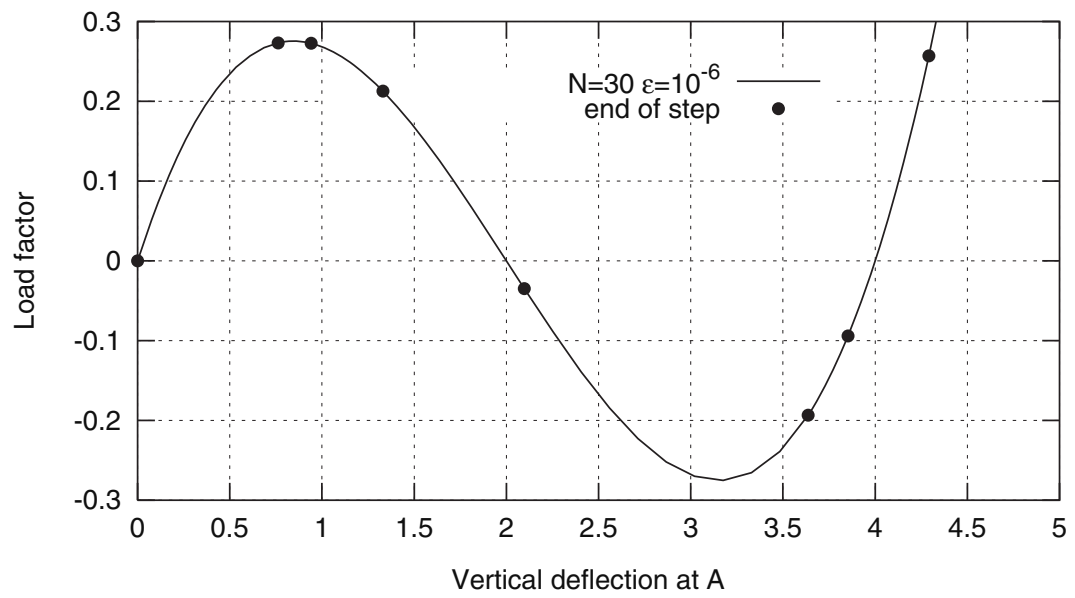

Figure 10. The first step ends after the bifurcation point and the fundamental branch is followed.

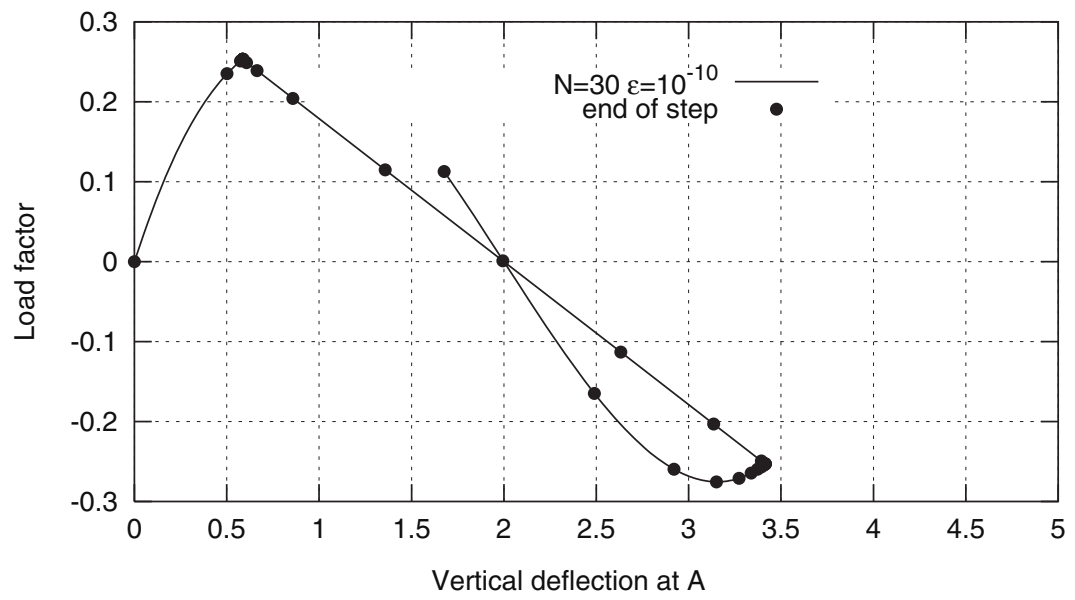

Figure 11. The first step ends before the bifurcation point and the algorithm turns onto the bifurcated branch.

the path, but only quasi-bifurcations as in the case of the simple imperfect models presented in Section 4. Here, the level of imperfection is unknown. It is certainly very small but, as we have seen before, it is sufficient for the series to have a finite radius of convergence which is given by the distance from the starting point to the bifurcation. That's why we had a shorter second step with an end point closed to the bifurcation (see Figures 3 and 4). This is also why it takes several steps to get away from the bifurcation, with a step length doubling. The level of imperfection can be inferred by reducing the tolerance error $\varepsilon_{R}$ as we did in Section 3 . Here it seems to be between $10^{-7}$ and $10^{-8}$. When the tolerance error $\varepsilon_{R}$ is below this level of imperfection (Figure 5), the end of the second step is before the bifurcation and the ANM 
continuation automatically makes very short steps to describe the very sharp turn onto the bifurcated branch. This should not be seen as a bad behaviour of the algorithm but, on the contrary, as a remarkable property of the algorithm to stay on a branch without jumping onto the opposite one.

\section{CONCLUSION}

In this paper, we have explained the behaviour of the ANM continuation when there is a bifurcation on a path. We should say 'when there is a quasi-bifurcation on a path' since it is the rule more than the exception because of inevitable small imperfections in the data of a finite element model. Pure or quasi-bifurcations generally do not matter for the classical incremental-iterative methods which continue to follow the fundamental branch by jumping over the bifurcation during one step. However, this does matter for the ANM continuation since the small imperfection can largely modify the radius of convergence of the power series representation of the path. With the criterion (4) for the continuation, the algorithm automatically reduces its step-length at the bifurcation in order to follow the path with the sharp turn, and it succeeds if the residual tolerance is small enough as compared to the level of imperfection.

We conclude with a few comments on the behaviour of the ANM continuation:

- The users of the ANM continuation know that a step accumulation is always a very good indicator that a singularity is announced on the path. All the bifurcations can easily be identified by the user without any specific tool.

- The turn onto the bifurcated branch is not a bad behaviour of the algorithm. On the contrary, it is a remarkable demonstration of the robustness of the continuation algorithm to stay on its branch even at a very sharp turn.

- By using a suitable very small tolerance $\varepsilon_{R}$, the algorithm could be seen as a way of switching to a bifurcated branch without specific tools. This is true, but it is unfortunately not efficient since it requires a lot of small steps to make the turn. We refer to References $[15,14]$ for efficient branch switching techniques.

- Having in mind the described phenomenon, the user can modify the parameter $\varepsilon_{R}$ and the order $N$ to jump efficiently over a quasi-bifurcation [11]. For instance, in the neighbourhood of a bifurcation, it is better to use low orders of truncature in order to have a step end which is far from the bifurcation after the jump and thus reduce the step accumulation.

\section{REFERENCES}

1. Allgower EL, Georg KG. Numerical Continuation Methods, An Introduction. Springer series in Computational Mathematics, vol. 13. Springer: Berlin, 1990.

2. Riks E. The application of Newton's method to the problem of elastic stability. Journal of Applied Mechanics, Transaction of ASME 1972; 39:1060-1066.

3. Ramm E. Strategies for tracing non-linear responses near limit points. In Non-linear Finite Element Analysis in Structural Mechanics, Stein E, Wunderlich W, Bathe KJ (eds). Springer: New York, 1981; 68-89.

4. Crisfield MA. Non-linear Finite Element Analysis of Solids and Structures, vol. 1. John Wiley and Sons: New York, 1991.

5. Cochelin B, Damil N, Potier-ferry M. The asymptotic numerical method: an efficient perturbation technique for nonlinear structural mechanics. Revue Européenne des éléments finis 1994; 3:281-297. 
6. Najah A, Cochelin B, Damil N, Potier-ferry M. A critical review of asymptotic numerical methods. Archives of Computational Methods in Engineering 1998; 5:31-50.

7. Zahrouni H, Cochelin B, Potier-ferry M. Asymptotic numerical method for shells with finite rotation. Computer Methods in Applied Mechanics and Engineering 1999; 175:271-285.

8. Cao HL, Potier-ferry M. An improved iterative method for large strain viscoplastic problems. International Journal for Numerical Methods in Engineering 1994; 44:155-176.

9. Elhage-Hussein A, Damil N, Potier-ferry M. A numerical continuation method based on Padé approximants. International Journal of Solids and Structures 2000; 37:6981-7001.

10. Cadou JM, Potier-ferry M, Cochelin B, Damil N. Asymptotic numerical method for stationary Navier-Stokes equation and with the Petrov-Galerkin formulation. International Journal for Numerical Methods in Engineering 2001; 50:825-845.

11. Baguet S. Stabilité des structures minces et sensibilité aux imperfections par la méthode asymptotique numérique. Ph.D. dissertation, Université de la Méditerranée, Marseille, October 2001.

12. Riks E. Some computational aspects of stability of nonlinear structures. Computer Methods in Applied Mechanics and Engineering 1984; 47:219-259.

13. Van Dyke M. Analysis and improvement of perturbations series. The Quarterly Journal of Mechanics and Applied Mathematics 1974; 27:423-450.

14. Vannucci P, Cochelin B, Damil N, Potier-Ferry M. An Asymptotic Numerical Method to compute bifurcating branches. International Journal for Numerical Methods in Engineering 1998; 41:1365-1389.

15. Kouhia R, Mikkola M. Tracing the equilibrium path beyond simple critical points. International Journal for Numerical Methods in Engineering 1989; 28:2923-2941. 